\title{
The anticancer effects of Cucurbitacin I inhibited cell growth of human non-small cell lung cancer through PI3K/AKT/p70S6K pathway
}

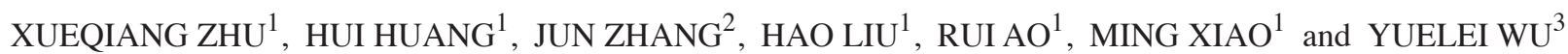 \\ ${ }^{1}$ Center of Oncology, Sichuan Provincial People's Hospital, Chengdu, Sichuan 610072; \\ ${ }^{2}$ Department of Oncology, The Third People's Hospital of Chengdu, Chengdu, Sichuan 610031; \\ ${ }^{3}$ Department of Gerontology, Chengdu First People's Hospital, Chengdu, Sichuan 610041, P.R. China
}

Received June 5, 2017; Accepted October 13, 2017

DOI: $10.3892 / \mathrm{mmr} .2017 .8141$

\begin{abstract}
The present study aimed to identify potential anticancer effects of Cucurbitacin I regulators on cell growth of human non-small cell lung cancer (NSCLC) and to explore their mechanism. The results indicated that the anticancer effects of Cucurbitacin I markedly attenuated cell proliferation, and induced apoptosis in NSCLC. Furthermore, Cucurbitacin I suppressed phosphatidylinositol-4,5-bisphosphate 3-kinase (PI3K), phosphorylation (p)-AKT and p-p70S6K pathway in NSCLC. Then, (PI3K) inhibitor increased anticancer effects of Cucurbitacin I on NSCLC. In conclusion, the present results indicated that Cucurbitacin I inhibited cell growth of human NSCLC through PI3K/AKT/p70S6K signaling pathway.
\end{abstract}

\section{Introduction}

Lung cancer is the malignant tumor with the highest fatality rate in the world. Meanwhile, non-small cell lung cancer (NSCLC) is the most common pathological type of lung cancer, accounting for $>70 \%$ of all lung cancer cases (1). The increasingly improved therapeutic means such as traditional surgery has greatly improved the prognosis and quality of life of lung cancer patients (1). However, intensive understanding of the molecular mechanism of lung cancer pathogenesis is lacking at present (2). Therefore, the existing early diagnosis target and anticancer therapy have limited effect on lung cancer patients (2). It is reported that $>50 \%$ NSCLC patients visiting for the first time have progressed into stage III or have metastases (2). As a result, they have been deprived of the opportunity of surgical treatment. Platinum-based combined chemotherapy regimen is considered as one of the most

Correspondence to: Mrs. Yuelei Wu, Department of Gerontology, Chengdu First People's Hospital, 18 Wanxiang North Road, Chengdu, Sichuan 610041, P.R. China

E-mail: ebwvms1640998@126.com

Key words: Cucurbitacin I, non-small cell lung cancer, phosphatidylinositol-4,5-bisphosphate 3-kinase, AKT, p70S6K effective methods for treating advanced NSCLC (3). However, tumor is a highly heterogeneous disease. It is associated with remarkably different sensitivity to platinum-based chemotherapeutics, even in lung cancer patients with same clinical stage or same pathological type (4).

As is suggested in research, the phosphatidylinositol-4,5-bisphosphate 3-kinase (PI3K)/AKT signaling pathway is abnormally activated in NSCLC (5). This has played an important role in tumor cell proliferation, apoptosis, survival and drug resistance (6). Cisplatin is the most common first-line chemotherapeutic in clinic (7). However, tumor resistance to cisplatin becomes increasingly common as treatment progresses, which has severely restricted its clinical effects (7). Cisplatin resistance is a complex process involving multiple mechanisms. Of them, continuous activation of the PI3K/AKT pathway or its components is one of the vital factors (6).

Chinese herbal medicine has been applied in treating disease for thousands of years in traditional medicine (8). For instance, Jinhuang powder and ichthammol ointment have the effects of softening hardness to dissipate stagnation, clearing heat and removing toxicity (8). Therefore, they have been used to treat non-suppurative acute mastitis and sebaceous cyst (9). In recent years, some scholars have studied the effects of Yishenguchong decoction, which is constituted by 11 kinds of Chinese herbs, on treating breast cancer (10). Cucurbitacin is a tetracyclic triterpenoid, which is abundant in cucurbitaceous plant (11). Data have indicated that cucurbitacin has anti-inflammatory, anti-bacterial and immunity boosting effects (10). Moreover, cucurbitacin is mainly employed in traditional medicine to assist in treating hepatitis or primary liver cancer. It has favorable therapeutic effects and moderate property (9). Cucurbitacin I is one of the Cucurbitacin family members with the richest content (12). At the same time, it is also one of the most extensively studied Cucurbitacin family members in terms of anticancer effects (8). Research in vivo and in vitro has suggested that Cucurbitacin I can inhibit cellular growth of multiple malignant tumors, such as liver cancer, gastric cancer, breast cancer and neuroblastoma (12). In addition, it can suppress their proliferation and apoptosis in the meantime of improving sensitivity of tumor cells to chemotherapeutics (12). In this study, we aimed to identify 
potential anticancer effects of Cucurbitacin I regulators cell growth of human NSCLC and to explore their mechanism.

\section{Materials and methods}

Cell culture. A549 lung adenocarcinoma were grown in complete DMEM containing 10\% FBS (both Gibco; Thermo Fisher Scientific, Inc., Waltham, MA, USA) at $37^{\circ} \mathrm{C}$ and $5 \% \mathrm{CO}_{2}$.

Cell proliferation assay and toxicity assay. Cell was treated with 0 (DMSO), 50, 100 and $200 \mathrm{nM}$ of Cucurbitacin I for 24,48 , and $72 \mathrm{~h}$, for $48 \mathrm{~h}$, and stained with MTT assay for $4 \mathrm{~h}$ at $37^{\circ} \mathrm{C}$. DMSO assay was used to dissolve for $20 \mathrm{~min}$ at $37^{\circ} \mathrm{C}$. Absorbance was measured using an MRX II microplate reader (Dynex, Chantilly, VA, USA) at $490 \mathrm{~nm}$.

We used LDH activity to explain cell cytotoxicity of Cucurbitacin I on NSCLC using LDH Release Assay kit (C0016; Beyotime Institute of Biotechnology, Haimen, China). Absorbance was measured using an MRX II microplate reader (Dynex) at $405 \mathrm{~nm}$.

Flow cytometry analysis. After treatment with Cucurbitacin I for $48 \mathrm{~h}$, cells washed with PBS three times. Cells were stained with all $5 \mu \mathrm{l}$ of Annexin V-FITC and PI (Sigma-Aldrich Co., St Louis, MO, USA) for $15 \mathrm{~min}$ at darkness. The apoptosis rate was analyzed by flow cytometry (BD FACSCanto II; BD Biosciences, San Diego, CA, USA).

Western blot analysis and caspase-3/9 activity. Protein samples from cells were prepared in RIPA buffer (Beyotime Institute of Biotechnology) and protein concentrations were measured using the BCA protein assay kit (Beyotime Institute of Biotechnology). The equal amounts $(40 \mu \mathrm{g})$ of protein were separated on $8-12 \%$ SDS gel and transferred onto PVDF membranes. Membranes were probed with the primary antibodies: PI3K (1:500), p-AKT (1:500), p-p70S6K (1:500) and GAPDH (1:500; all Santa Cruz, CA, USA) at $4^{\circ} \mathrm{C}$ overnight. After incubating with HRP-conjugated secondary antibodies goat anti-rabbit IgG, protein bands were detected using an enhanced chemiluminescence detection kit (ThermoFisher Scientific, Inc.). Equal amounts protein also was used to measure caspsae-3/9 activity using caspsae-3/9 activity kits (Beyotime Institute of Biotechnology).

Statistical analysis. Data were presented as the mean \pm standard error. Statistical differences were analyzed using the one-way analysis of variance (ANOVA) with the Tukey's test. $\mathrm{P}<0.05$ was considered to indicate a statistically significant difference.

\section{Results}

Cucurbitacin I attenuated cell proliferation of NSCLC. To investigate whether the anticancer of Cucurbitacin I on NSCLC, A549 cell was treated with Cucurbitacin I, cell proliferation and LDH activity were measured. The structural formula of Cucurbitacin I was showed at Fig. 1A. As showed in Fig. 1B, C, Cucurbitacin I attenuated cell proliferation and increased LDH activity in A549 cell, compared with control group.
Cucurbitacin I induced apoptosis of NSCLC. Next, Cucurbitacin I induced apoptosis, and promoted capase-3/9 activity in A549 cell, compared with control group (Fig. 2).

Cucurbitacin I suppressed PI3K/AKT/p70S6K pathway of NSCLC. To identify the PI3K/AKT/p70S6K pathway mediator involved in the anticancer effects of Cucurbitacin I on NSCLC, we examined the changes of PI3K/AKT/p70S6K pathway. Cucurbitacin I suppressed PI3K, p-AKT and p-p70S6K protein expression, compared with control group (Fig. 3).

Suppression of PI3K increased the anticancer effects of Cucurbitacin I on PI3K/AKT/p70S6K pathway of NSCLC. To validate the downregulation of $\mathrm{PI} 3 \mathrm{~K} / \mathrm{AKT} / \mathrm{p} 70 \mathrm{~S} 6 \mathrm{~K}$ pathway by PI3K inhibitor (LY294002; $100 \mathrm{nM}$ ) affects the anticancer effects of Cucurbitacin I on NSCLC. PI3K inhibitor suppressed PI3K, p-AKT and p-p70S6K protein expression, compared with control group. Taken together, PI3K/AKT/p70S6K pathway maybe mediates the anticancer effects of Cucurbitacin I on NSCLC (Fig. 4).

Suppression of PI3K increased the anticancer effects of Cucurbitacin I on cell proliferation of NSCLC. Fig. 5 showed that the suppression of PI3K increased the anticancer effects of Cucurbitacin I on the inhibition of cell proliferation and the activation of LDH activity of NSCLC, compared with only Cucurbitacin I treatment group (Fig. 5).

Suppression of PI3K increased the anticancer effects of Cucurbitacin I on apoptosis of NSCLC. Meanwhile, the suppression of PI3K increased the anticancer effects of Cucurbitacin I on the promotion of apoptosis and caspase-3/9 activity of NSCLC, compared with only Cucurbitacin I treatment group (Fig. 6). Overall, these data suggest that Cucurbitacin I induced apoptotic in NSCLC involve inhibition of $\mathrm{PI} 3 \mathrm{~K} / \mathrm{AKT} / \mathrm{p} 70 \mathrm{~S} 6 \mathrm{~K}$ pathway.

Suppression of p70S6K reduced the anticancer effects of Cucurbitacin I on PI3K/AKT/p70S6K pathway of NSCLC. We next explored whether p70S6K is involved in the anticancer effects of Cucurbitacin I on cell proliferation of NSCLC. As showed in Fig. 7, p70S6K inhibitor (LY-2584702 hydrochloride, $2 \mathrm{nM}$ ) suppressed the protein expression of p-p70S6K in NSCLC by Cucurbitacin I, compared with only Cucurbitacin I treatment group (Fig. 7).

Suppression of p70S6K reduced the anticancer effects of Cucurbitacin I on cell proliferation of NSCLC. The suppression of p70S6K increased the anticancer effects of Cucurbitacin I on the inhibition of cell proliferation and the activation of LDH activity of NSCLC, compared with only Cucurbitacin I treatment group (Fig. 8).

Suppression of p70S6K reduced the anticancer effects of Cucurbitacin I on apoptosis of NSCLC. Moreover, the suppression of p70S6K increased the anticancer effects of Cucurbitacin I on the promotion of apoptosis and caspase-3/9 activity of NSCLC, compared with only Cucurbitacin I treatment group (Fig. 9). 

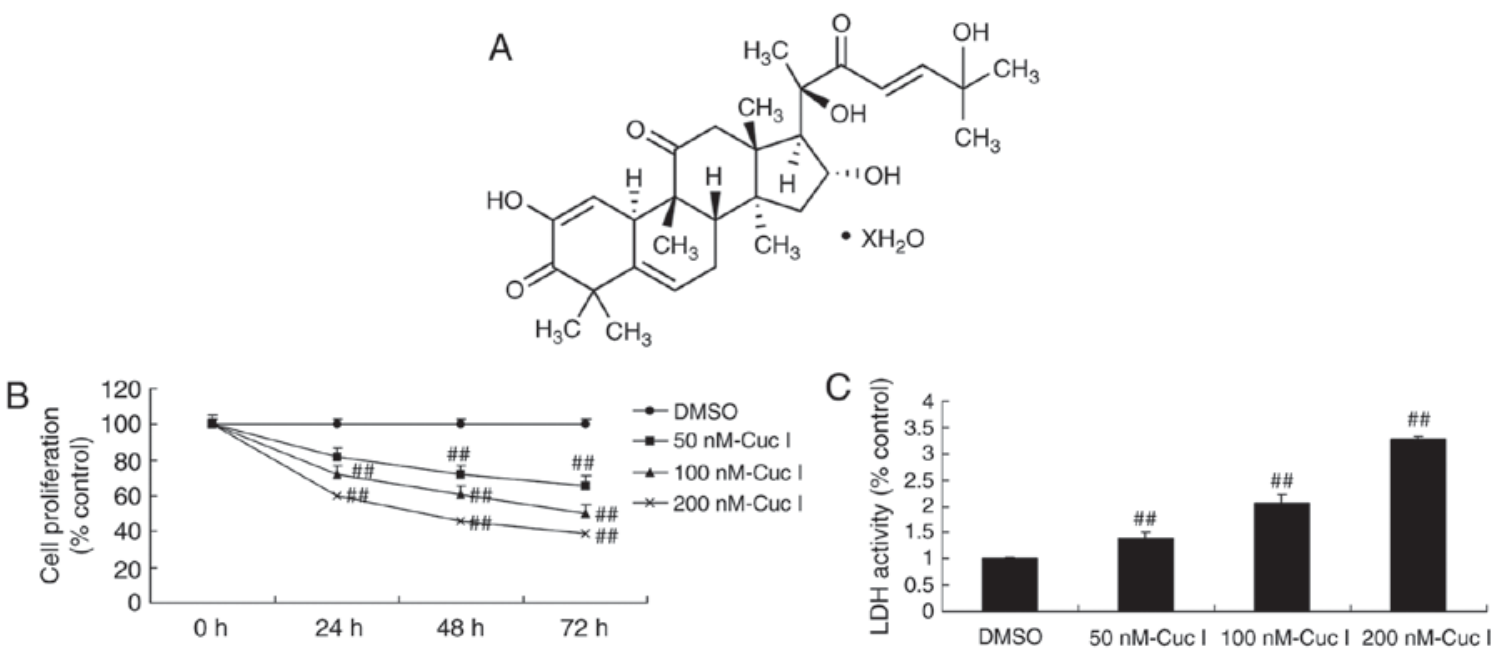

Figure 1. Cucurbitacin I attenuated cell proliferation of NSCLC. The structural formula of Cucurbitacin I (A), cell proliferation (B) and LDH activity (C).

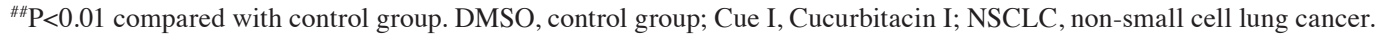
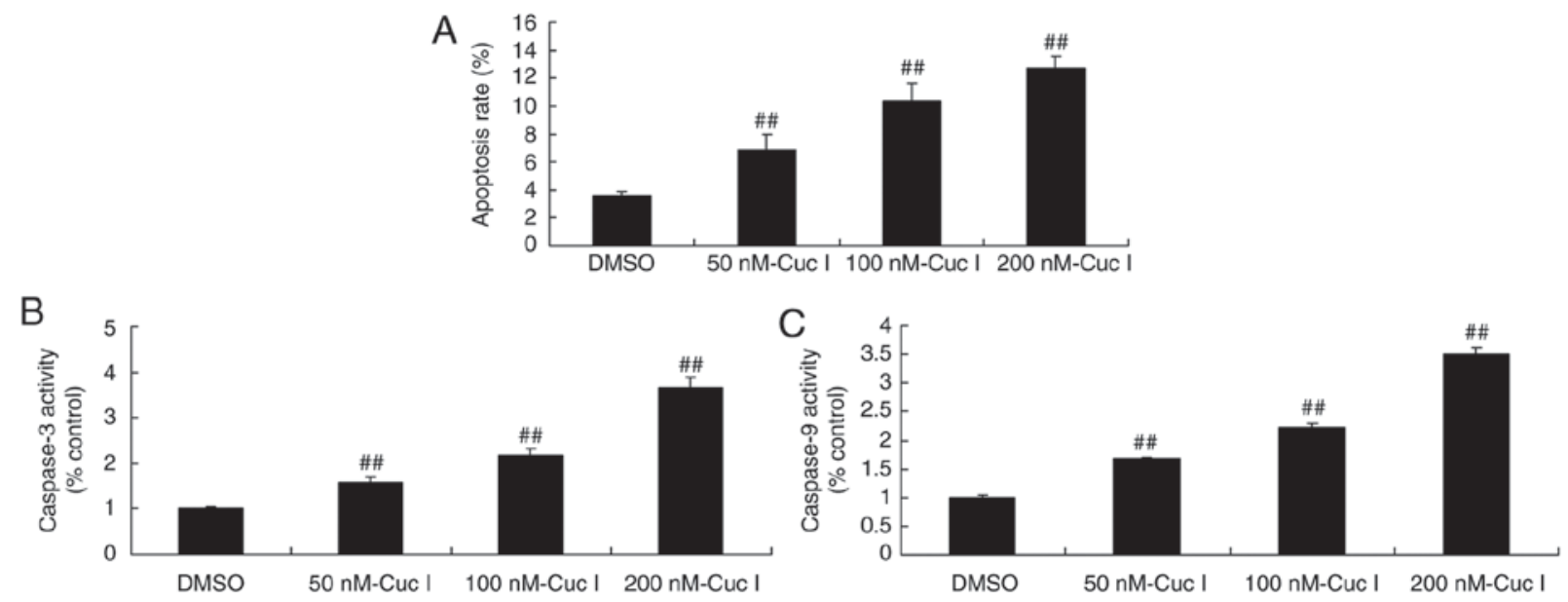

Figure 2. Cucurbitacin I induced apoptosis of NSCLC. Apoptosis rate (A), caspase-3 activity (B) and caspase-9 activity (C). NSCLC, non-small cell lung cancer; DMSO, control group; Cue I, Cucurbitacin I. ${ }^{\# \#} \mathrm{P}<0.01$ compared with control group.
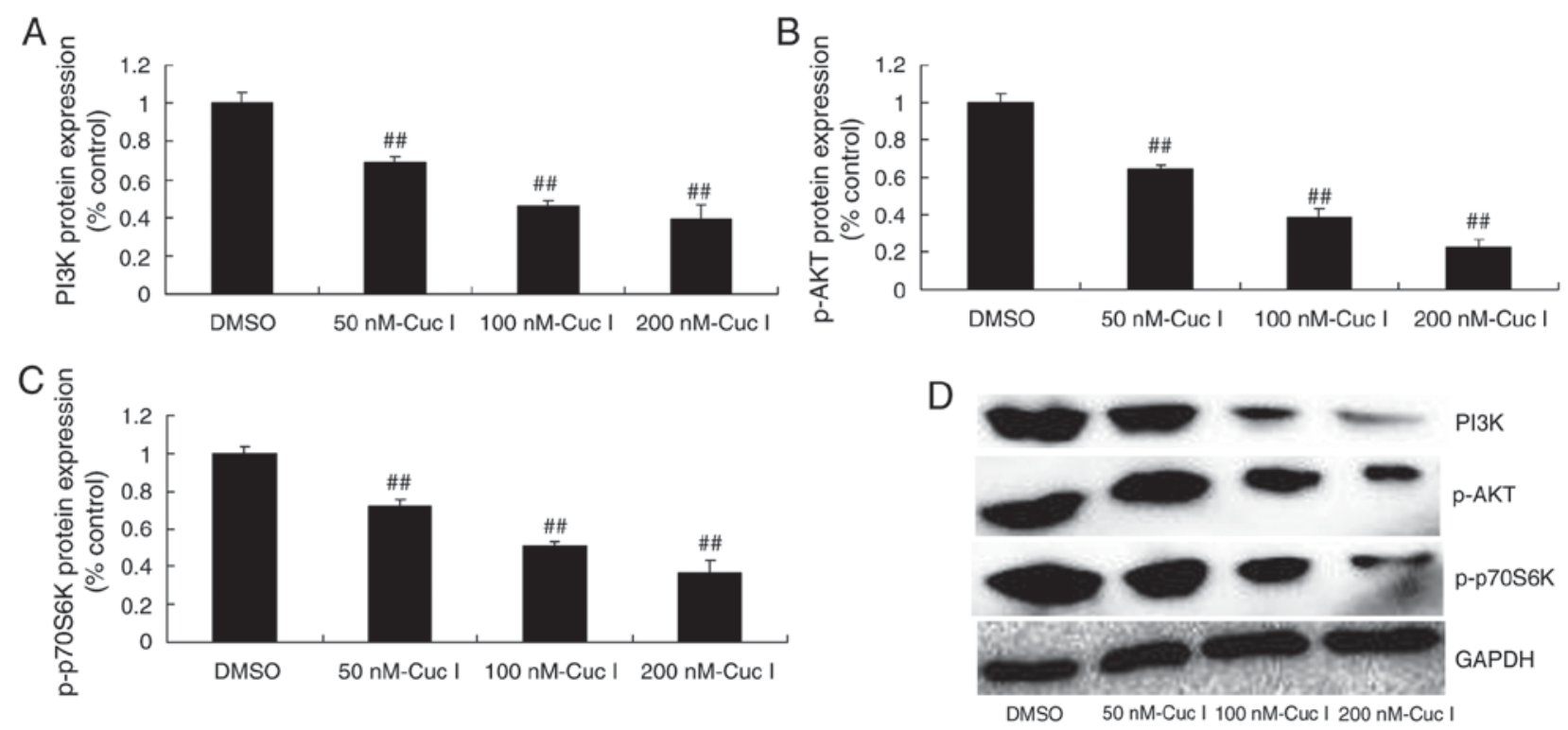

Figure 3. Cucurbitacin I suppressed PI3K/AKT/ p70S6K pathway of NSCLC. PI3K, p-AKT and p-p70S6K protein expression by statistical analysis (A-C), and western blot analysis (D). ${ }^{\# \#} \mathrm{P}<0.01$ compared with control group. DMSO, control group; Cue I, Cucurbitacin I. 

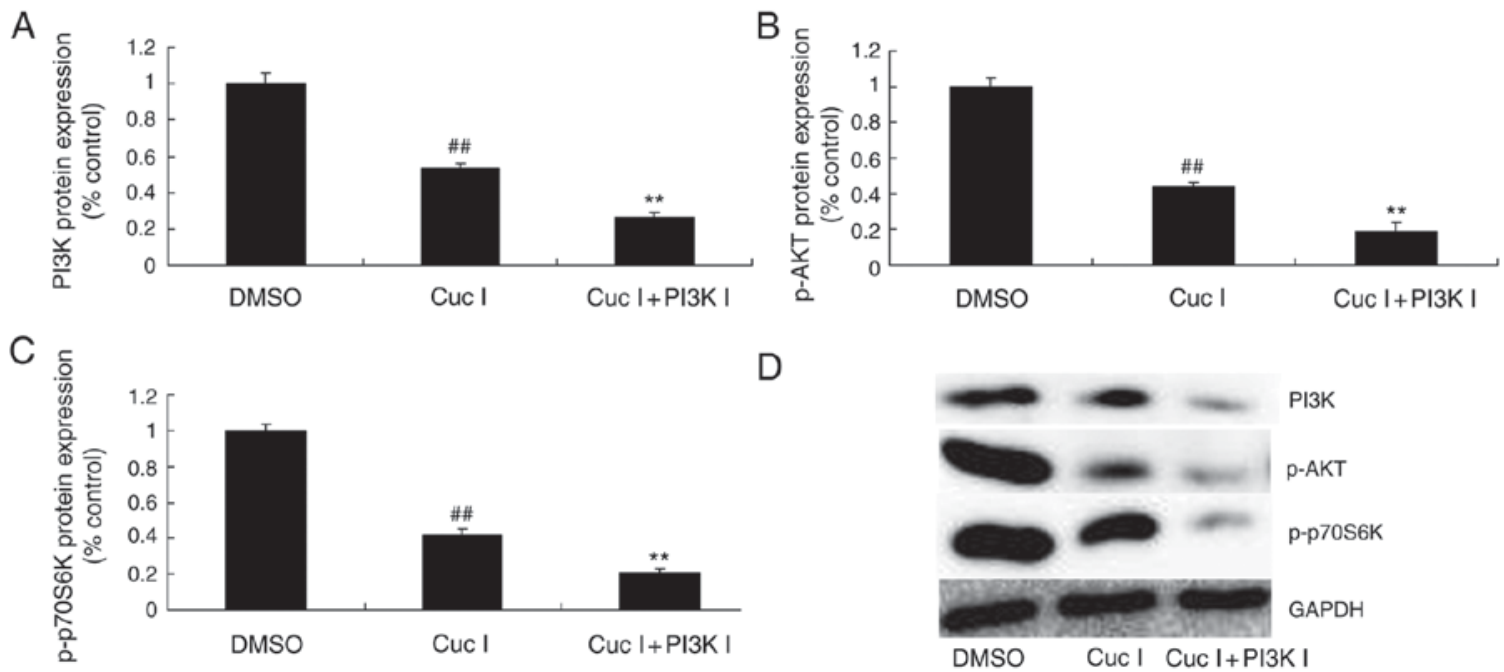

Figure 4. The suppression of PI3K increased the anticancer effects of Cucurbitacin I on PI3K/AKT/p70S6K pathway of NSCLC. PI3K, p-AKT and p-p70S6K protein expression by statistical analysis (A-C), and western blot analysis (D). ${ }^{\# \#} \mathrm{P}<0.01$ compared with control group, ${ }^{* *} \mathrm{P}<0.01$ compared with Cue I group. DMSO, control group; Cue I, Cucurbitacin I; PI3K, phosphatidylinositol-4,5-bisphosphate 3-kinase.
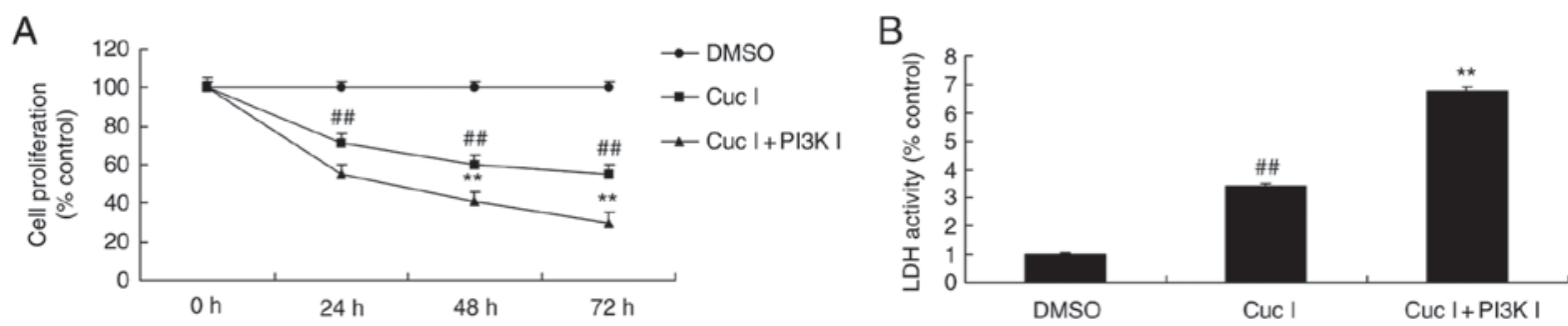

Figure 5. The suppression of PI3K increased the anticancer effects of Cucurbitacin I on cell proliferation of NSCLC. Cell proliferation (A) and LDH activity (B). ${ }^{\# \prime} \mathrm{P}<0.01$ compared with control group, ${ }^{* *} \mathrm{P}<0.01$ compared with Cue I group. DMSO, control group; Cue I, Cucurbitacin I; PI3K, phosphatidylinositol-4,5-bisphosphate 3-kinase.
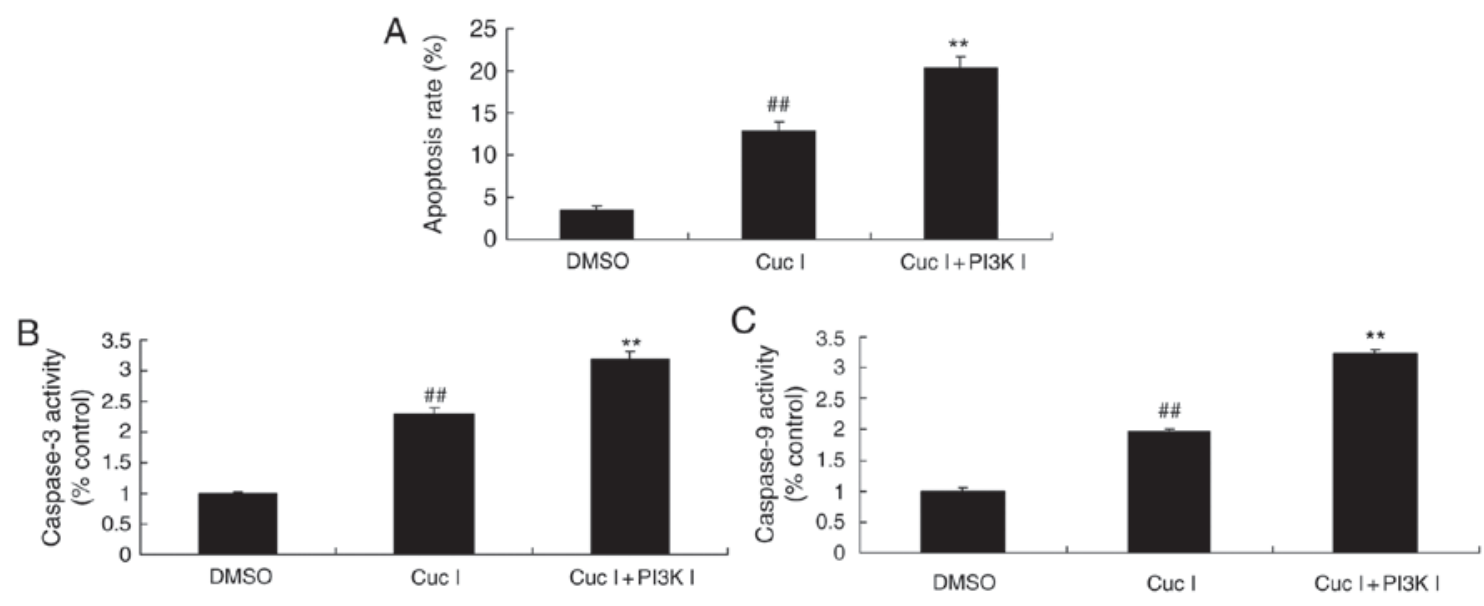

Figure 6. The suppression of PI3K increased the anticancer effects of Cucurbitacin I on apoptosis of NSCLC. Apoptosis rate (A), caspase-3 activity (B) and caspase-9 activity (C). ${ }^{\# \#} \mathrm{P}<0.01$ compared with control group, ${ }^{* *} \mathrm{P}<0.01$ compared with Cue I group. NSCLC, non-small cell lung cancer; DMSO, control group; Cue I, Cucurbitacin I; PI3K, phosphatidylinositol-4,5-bisphosphate 3-kinase.

\section{Discussion}

Lung cancer is a severe disease affecting human life. The world has witnessed over 1.1 million of patients dying of lung cancer, including $80 \%$ NSCLC patients (4). Surgical resection remains the preferred method for treating NSCLC at present (13). However, most patients have developed into moderate and advanced stage at diagnosis, which has deprived them of the optimal surgical timing. Therefore, they can only extend their life through chemotherapy or radiotherapy (13). In addition, most patients undergoing surgery requires adjuvant chemotherapy (14). Consequently, chemotherapy is an 
A

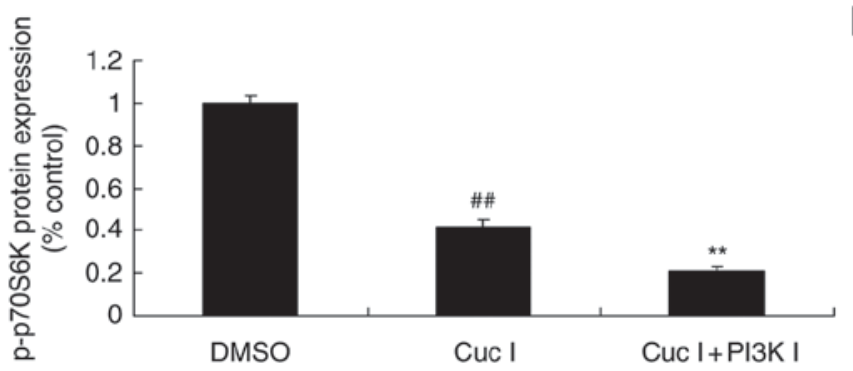

B

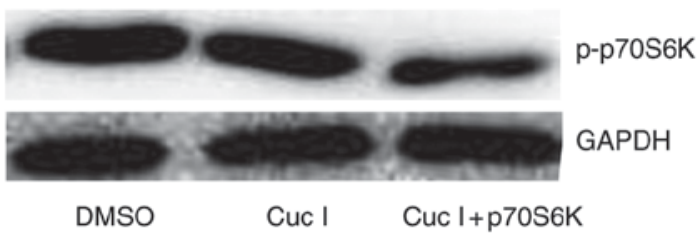

Figure 7. The inhibition of p70S6K reduced the anticancer effects of Cucurbitacin I on p70S6K pathway of NSCLC. p-p70S6K protein expression by statistical analysis (A), and western blot analysis (B). ${ }^{\# \#} \mathrm{P}<0.01$ compared with control group, ${ }^{* *} \mathrm{P}<0.01$ compared with Cue I group. NSCLC, non-small cell lung cancer; DMSO, control group; Cue I, Cucurbitacin I.
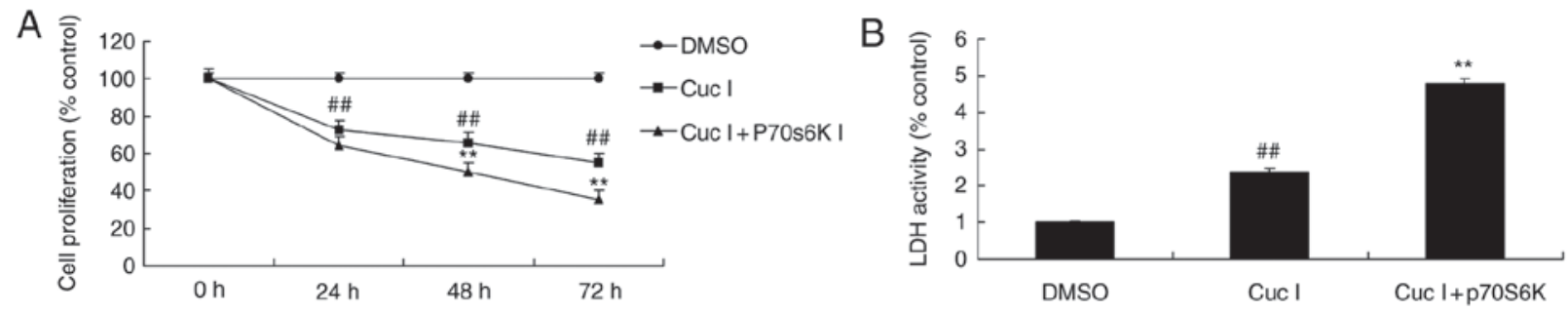

Figure 8. The inhibition of p70S6K reduced the anticancer effects of Cucurbitacin I on cell proliferation of NSCLC. Cell proliferation (A) and LDH activity (B). ${ }^{\# \#} \mathrm{P}<0.01$ compared with control group, ${ }^{* *} \mathrm{P}<0.01$ compared with Cue I group. DMSO, control group; Cue I, 1Cucurbitacin I; NSCLC, non-small cell lung cancer.
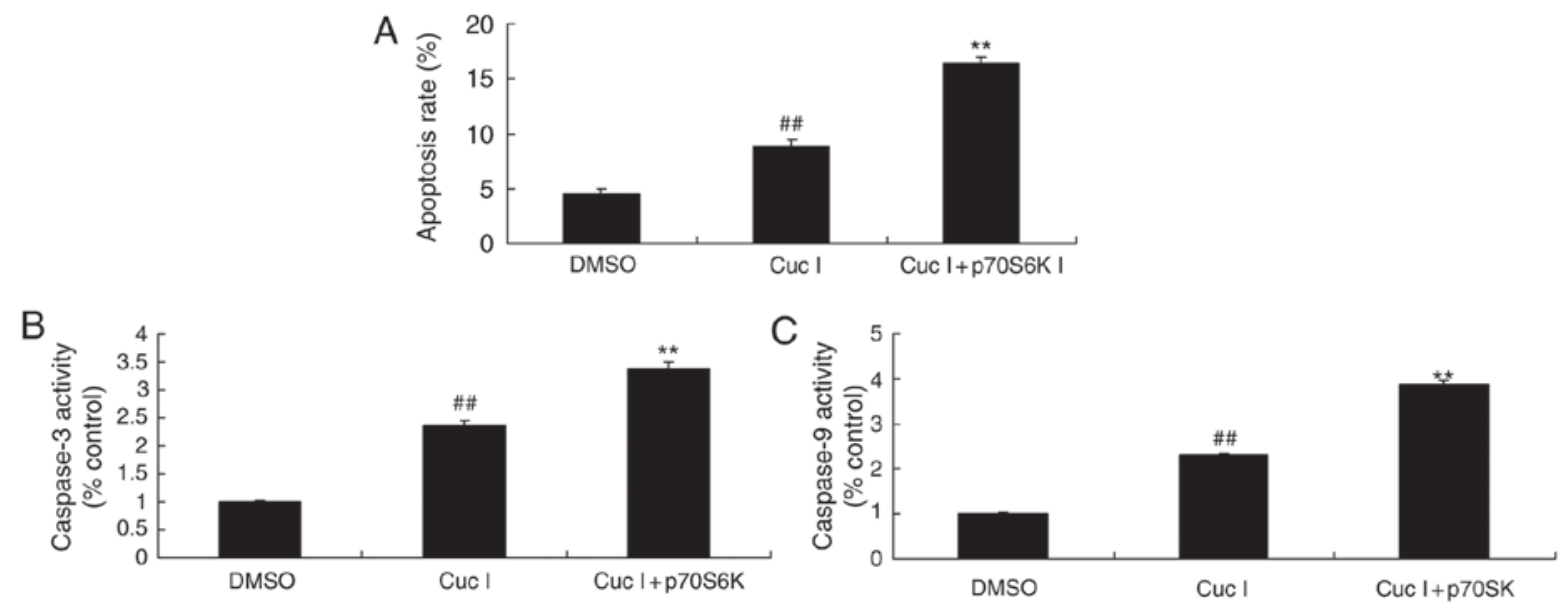

Figure 9. The inhibition of p70S6K reduced the anticancer effects of Cucurbitacin I on apoptosis of NSCLC. Apoptosis rate (A), caspase-3 activity (B) and caspase-9 activity $(\mathrm{C}) .{ }^{\# \#} \mathrm{P}<0.01$ compared with control group, ${ }^{* *} \mathrm{P}<0.01$ compared with Cue I group. NSCLC, non-small cell lung cancer; DMSO, control group; Cue I, Cucurbitacin I.

indispensable means for treating tumor and delaying patients' life span (15). The current study indicated Cucurbitacin I markedly attenuated cell proliferation in NSCLC. However, we only used A549 cell, which is a limitation of our study. So,further studies will be conducted with more CSCLC cell lines in order to confirm the study's results.

Genotype difference of NSCLC is suggested to be partly correlated with body sensitivity to platinum-based chemotherapeutics (16). Platinum-based chemotherapeutics, such as cisplatin and carboplatin, are one of the most extensively used anticancer chemotherapeutics with the most definite efficacy (17). Moreover, they show strong cytotoxicity (16). Platinum-based drugs will bind with intranuclear DNA after entering the cell nucleus. They can lead to irreversible DNA damage through forming the platinum-DNA complex (18). Meanwhile, they can also induce cell apoptosis and thus exert their anticancer effects (16). The intracellular DNA damage repair mechanism allows cells to recruit DNA damage repair-related factors to repair the DNA damage induced by platinum-based chemotherapeutics (18). Therefore, expression and function of regulatory factors during DNA repair process are considered to play a determining role in tumor sensitivity to platinum-based chemotherapeutics (17). The results of the present study indicated that Cucurbitacin I markedly induced apoptosis in NSCLC. Ishdorj et al demonstrates that Cucurbitacin-I activates of apoptosis and cell cycle 
arrest, leading to increased VEGF expression in B leukemic cells (11).

Exon point mutation of gene PI3K is found in multiple human tumor cells, such as colorectal cancer, breast cancer, brain cancer, liver cancer, gastric cancer, ovarian cancer and lung cancer. About 3\% NSCLC patients have PI3K mutation in tumor cells (19). The PI3K/Akt signal transduction pathway plays a vital role in growth factor-mediated cell survival (20). Research has indicated that, dysfunction of the $\mathrm{PI} 3 \mathrm{~K} / \mathrm{Akt} / \mathrm{mTOR}$ pathway may play a crucial role in lung cancer formation (19). As has been reported, several cell proliferation signals produced by the binding of transmembrane receptor with ligand can activate the transduction of PI3K/Akt/mTOR signal (20). This is closely associated with the proliferation and survival of NSCLC (7). Furthermore, out showed that Cucurbitacin I suppressed PI3K and p-AKT protein expression in NSCLC. Johnson et al indicate that Cucurbitacin I blocks cerebrospinal fluid through Akt signal (21).

In recent years, the role of $\mathrm{PI} 3 \mathrm{~K} / \mathrm{Akt} / \mathrm{m}$ TOR signaling pathway in human tumor has attracted extensive attention (22). Activation of PI3K/Akt/m TOR signaling pathway is quite common in the genesis of human tumor (5). Such pathway can promote cancer genesis through multiple mechanisms, including gene mutation, decreased expression of tumor suppressor gene PTEN, PI3K mutation or amplification, Akt mutation or amplification, and activation of oncogene receptor (23). Moreover, activation of all pathway components is a factor indicating poor prognosis for numerous tumors, which can induce treatment resistance (20). Inhibiting such pathway can reverse the resistance, and improve the chemotherapy and radiotherapy effects both in vivo and in vitro (23). Consequently, it is necessary to intensively study the precise mechanism of that pathway (22). Our study showed that PI3K inhibitor increased anticancer effects of Cucurbitacin I on NSCLC. Premkumar et al reported that Cucurbitacin I induces defects in cell cycle progression and promotes ABT-737 cell death of cell death through PI3K/Akt (24).

P70s6k is one of the direct substrates of p-mTOR protein, which can be phosphorylated by p-mTOR protein (25). The phosphorylated p70s6k can further phosphorylate the $40 \mathrm{~S}$ ribosomal subunit of protein S6, so as to enhance mRNAs translation (26). In this way, the ribosome, initiation factor and elongation factor can be produced, thus promoting tumor cell diffusion (26). Research has suggested that $\mathrm{p} 70 \mathrm{~s} 6 \mathrm{k}$ protein is closely associated with tumors such as digestive system neoplasms, ovarian cancer, breast cancer, hemangiomas and parotid adenocarcinoma. P-mTOR/p70s6k signaling pathway can regulate expression of downstream genes, thus affecting protein synthesis, cell proliferation and apoptosis (27). Moreover, it is closely related to the genesis, growth, metastasis and resistance of malignant tumor. Thus, it has become a hotspot of current tumor research (25). We found that Cucurbitacin I suppressed p-p70S6K pathway in NSCLC. Yuan et al showed that Cucurbitacin I induces protective autophagy through p70S6K pathway in glioblastoma in vitro and in vivo (12).

In conclusion, the present study demonstrated that anticancer effects of Cucurbitacin I inhibited cell growth of human NSCLC through PI3K/AKT/p70S6K pathway.
Therefore, Cucurbitacin I may present a novel therapeutic treatment for NSCLC.

\section{References}

1. Yang JC, Ahn MJ, Kim DW, Ramalingam SS, Sequist LV, Su WC, Kim SW, Kim JH, Planchard D, Felip E, et al: Osimertinib in pretreated T790M-positive advanced non-small-cell lung cancer: AURA study phase II extension component. J Clin Oncol 35: 1288-1296, 2017.

2. Crawford J, Swanson P, Schwarzenberger P, Sandler A, Prager D, Zhang K, Freeman DJ, Johnson CW, Krishnan K and Johnson D: A phase 2 randomized trial of paclitaxel and carboplatin with or without panitumumab for first-line treatment of advanced non-small-cell lung cancer. J Thorac Oncol 8: 1510-1518, 2013.

3. Kubota K, Ichinose Y, Scagliotti G, Spigel D, Kim JH, Shinkai T, Takeda K, Kim SW, Hsia TC, Li RK, et al: Phase III study (MONET1) of motesanib plus carboplatin/paclitaxel in patients with advanced nonsquamous nonsmall-cell lung cancer (NSCLC): Asian subgroup analysis. Ann Oncol 25: 529-536, 2014.

4. Liang J, Bi N, Wu S, Chen M, Lv C, Zhao L, Shi A, Jiang W, $\mathrm{Xu}$ Y, Zhou Z, et al: Etoposide and cisplatin versus paclitaxel and carboplatin with concurrent thoracic radiotherapy in unresectable stage III non-small cell lung cancer: A multicenter randomized phase III trial. Ann Oncol 28: 777-783, 2017.

5. Wang B, Jiang H, Wang L, Chen X, Wu K, Zhang S, Ma S and Xia B: Increased MIR31HG lncRNA expression increases gefitinib resistance in non-small cell lung cancer cell lines through the EGFR/PI3K/AKT signaling pathway. Oncol Lett 13: 3494-3500, 2017

6. Chao W, Deng JS, Li PY, Liang YC and Huang GJ: 3,4-Dihydroxybenzalactone suppresses human non-smallcelllung carcinoma cells metastasis via suppression of epithelial to mesenchymal transition, ROS-Mediated PI3K/AKT/MAPK/MMP and NFKB signaling pathways. Molecules 22: pii: E537, 2017.

7. Pérez-Ramírez C,Cañadas-Garre M, Molina MÁ, Faus-Dáder MJ and Calleja-Hernández MÁ: PTEN and PI3K/AKT in non-small-cell lung cancer. Pharmacogenomics 16: 1843-1862, 2015.

8. Wu Y, Chen H, Li R, Wang X, Li H, Xin J, Liu Z, Wu S, Jiang W and Zhu L: Cucurbitacin-I induces hypertrophy in H9c2 cardiomyoblasts through activation of autophagy via MEK/ERK1/2 signaling pathway. Toxicol Lett 264: 87-98, 2016.

9. Song J, Liu H, Li Z, Yang C and Wang C: Cucurbitacin I inhibits cell migration and invasion and enhances chemosensitivity in colon cancer. Oncol Rep 33: 1867-1871, 2015.

10. Qi J, Xia G, Huang CR, Wang JX and Zhang J: JSI-124 (Cucurbitacin I) inhibits tumor angiogenesis of human breast cancer through reduction of STAT3 phosphorylation. Am J Chin Med 43: 337-347, 2015.

11. Ishdorj G, Johnston JB and Gibson SB: Cucurbitacin-I (JSI-124) activates the JNK/c-Jun signaling pathway independent of apoptosis and cell cycle arrest in B leukemic cells. BMC Cancer 11: 268, 2011

12. Yuan G, Yan SF, Xue H, Zhang P, Sun JT and Li G: Cucurbitacin I induces protective autophagy in glioblastoma in vitro and in vivo. J Biol Chem 289: 10607-10619, 2014.

13. Sharma N, Pennell N, Nickolich M, Halmos B, Ma P, Mekhail T, Fu P and Dowlati A: Phase II trial of sorafenib in conjunction with chemotherapy and as maintenance therapy in extensive-stage small cell lung cancer. Invest New Drugs 32: 362-368, 2014.

14. Heigener DF, Pereira JR, Felip E, Mazal J, Manzyuk L, Tan EH, Merimsky O, Sarholz B, Esser R and Gatzemeier U: Weekly and every 2 weeks cetuximab maintenance therapy after platinum-based chemotherapy plus cetuximab as first-line treatment for non-small cell lung cancer: Randomized non-comparative phase IIIb NEXT trial. Target Oncol 10: 255-265, 2015.

15. Larkins E, Scepura B, Blumenthal GM, Bloomquist E, Tang S, Biable M, Kluetz P, Keegan P and Pazdur R: U.S. Food and drug administration approval summary: ramucirumab for the treatment of metastatic non-small cell lung cancer following disease progression on or after platinum-based chemotherapy. Oncologist 20: 1320-1325, 2015. 
16. Li L, Zhu T, Gao YF, Zheng W, Wang CJ, Xiao L, Huang MS, Yin JY, Zhou HH and Liu ZQ: Targeting DNA damage response in the radio(Chemo)therapy of non-small cell lung cancer. Int J Mol Sci 17: pii: E839, 2016

17. Shen H, Wang L, Zhang J, Dong W, Zhang T, Ni Y, Cao H, Wang K, Li Y, Wang Y and Du J: ARRB1 enhances the chemosensitivity of lung cancer through the mediation of DNA damage response. Oncol Rep 37: 761-767, 2017.

18. Liu D, Wu C, Jiao Y, Hou L, Lu D, Zheng H, Chen C, Qian J, Fei K and Su B: WEE1 kinase polymorphism as a predictive biomarker for efficacy of platinum-gemcitabine doublet chemotherapy in advanced non-small cell lung cancer patients. Sci Rep 5: 11114, 2015.

19. Chou PY, Huang GJ, Pan $\mathrm{CH}$, Chien YC, Chen YY, Wu CH, Sheu MJ and Cheng HC: Trilinolein inhibits proliferation of human non-small cell lung carcinoma A549 through the modulation of PI3K/Akt pathway. Am J Chin Med 39: 803-815, 2011.

20. Sun Z, Wang Z, Liu X and Wang D: New development of inhibitors targeting the PI3K/AKT/mTOR pathway in personalized treatment of non-small-cell lung cancer. Anticancer Drugs 26: $1-14,2015$.

21. Johnson MD, O'Connell MJ and Walter K: Cucurbitacin I blocks cerebrospinal fluid and platelet derived growth factor-BB stimulation of leptomeningeal and meningioma DNA synthesis. BMC Complement Altern Med 13: 303, 2013.

22. Fumarola C, Bonelli MA, Petronini PG and Alfieri RR Targeting PI3K/AKT/mTOR pathway in non small cell lung cancer. Biochem Pharmacol 90: 197-207, 2014.
23. Park C, Hong SH, Kim GY and Choi YH: So-Cheong-Ryong-Tang induces apoptosis through activation of the intrinsic and extrinsic apoptosis pathways, and inhibition of the PI3K/Akt signaling pathway in non-small-cell lung cancer A549 cells. BMC Complement Altern Med 15: 113, 2015.

24. Premkumar DR, Jane EP and Pollack IF: Cucurbitacin-I inhibits Aurora kinase A, Aurora kinase B and survivin, induces defects in cell cycle progression and promotes ABT-737-induced cell death in a caspase-independent manner in malignant human glioma cells. Cancer Biol Ther 16: 233-243, 2015.

25. In JK, Kim JK, Oh JS and Seo DW: 5-Caffeoylquinic acid inhibits invasion of non-small cell lung cancer cells through the inactivation of p70S6K and Akt activity: Involvement of p53 in differential regulation of signaling pathways. Int J Oncol 48: 1907-1912, 2016.

26. Dhar R and Basu A: Constitutive activation of p70 S6 kinase is associated with intrinsic resistance to cisplatin. Int J Oncol 32: 1133-1137, 2008

27. Dong S, Zhang XC, Cheng H, Zhu JQ, Chen ZH, Zhang YF, $\mathrm{Xie} \mathrm{Z}$ and $\mathrm{Wu} \mathrm{YL}$ : Everolimus synergizes with gefitinib in non-small-cell lung cancer cell lines resistant to epidermal growth factor receptor tyrosine kinase inhibitors. Cancer Chemother Pharmacol 70: 707-716, 2012. 